\title{
REMARKS ON DETERMINANT LINE BUNDLES, CHERN-SIMONS FORMS AND INVARIANTS
}

\author{
JOHAN L. DUPONT and FLEMMING LINDBLAD JOHANSEN*
}

\begin{abstract}
We study generalized determinant line bundles for families of principal bundles and connections. We explore the connections of this line bundle and give conditions for the uniqueness of such. Furthermore we construct for families of bundles and connections over manifolds with boundary, a generalized Chern-Simons invariant as a section of a determinant line bundle.
\end{abstract}

\section{Introduction}

Determinant line bundles were first constructed for families of Riemann surfaces by D. Quillen in [13]. This was generalized to higher dimensions by J. M. Bismut and D. Freed (see e.g. [4], [10] and [11]), and in [8] it was used by X. Dai and D. Freed to define a generalization of the Atiyah-Patodi-Singer $\eta$-invariant for families of Riemannian manifolds with boundary as a section of the inverse line bundle associated to the family of boundaries. All these constructions were analytical ones involving kernel/cokernels of differential operators.

In this paper we shall study a geometric construction of determinant line bundles going back to T. R. Ramadas, I. M. Singer and J. Weitsman (see [14]) for the case of families of connections in trivial $S U(2)$ bundles over closed surfaces and for more general families of principal bundles to the work by L. Bonora, P. Cotta-Ramusino, M. Rinaldi and J. Stasheff (see [1]; see also Brylinski [2] and [3]).

The construction in section 1 requires a smooth, closed, even-dimensional manifold $X$ and a family of principal bundles over $X$, each with a connection, which constitute a fibre bundle over $Z$. Together with an invariant polynomial this enables us to calculate transition functions of a line bundle $\mathscr{L}$ over $Z$. In section 2 we explore the connections of this line bundle. If the family $A_{z}$ of connections can be extended into a connection in the $Z$-direction also, there is a canonical connection of the line bundle. We describe these connections and determine when they are independent of the extensions.

\footnotetext{
* Work supported in parts by Statens Naturvidenskabelige Forskningsråd, Denmark.

Received August 31, 1999.
} 
Section 3 deals with generalizations of the Chern-Simons invariant. This was originally defined by S. S. Chern and J. Simons in [7] (see also CheegerSimons [6] or [9]) for a single connection in a principal bundle over a closed manifold. In analogy with the construction by Freed and Dai of an $\eta$-invariant section of the determinant line bundle mentioned above, we define, for a family of bundles and connections over a family of manifolds with boundary, a natural section cs ("Chern-Simons section") of the inverse line bundle which is an exponentiated version of a generalized Chern-Simons invariant. Finally in Section 4 we make a calculation of the line bundle, its connection and ChernSimons section in the simplest possible case of flat connections over genus $g$ handle bodies. It appears that the connection in this case is flat and that the section is parallel.

The authors would like to thank the referee for valuable comments and corrections.

Notation. The sign convention in this paper has been chosen in accordance with Bott and Tu (see [5]). If $\alpha$ is a form on $X$ and $\beta$ is a form on $Y$ then integration over the fibre in $X \times Y \rightarrow X$ is defined as

$$
\int_{Y} \alpha \wedge \beta=\left(\int_{Y} \beta\right) \alpha .
$$

This implies that differentiation commutes with integration over the fibre, i.e. $d_{X} \int_{Y} \alpha \wedge \beta=\int_{Y}\left(d_{X} \alpha\right) \wedge \beta$.

\section{Geometric Construction of a Determinant Line Bundle}

First let us recall the construction of a line bundle as in [1] for the following data.

GeOmetric DAta 1.1.

(1) A smooth, closed, oriented manifold $X$ of dimension $2 k-2$

(2) A Lie group $G$, a principal $G$-bundle $\mathscr{P} \rightarrow X$ and an invariant polynomial $P \in I_{0}^{k}(G)$

(3) A fixed connection $A_{0}$ of $\mathscr{P}$

(4) A fibre bundle $\mathscr{P} \rightarrow E \rightarrow Z$, where each fibre has a connection and the transition functions are gauge transformations homotopic to the identity

A few explanatory remarks here would seem to be in order:

i) The set of invariant polynomials of degree $k$, that is, of the $G$-invariant symmetric, multilinear functions in $k$ variables on the Lie algebra $g$, is 
denoted $I^{k}(G)$; the subset $I_{0}^{k}(G)$ of $I^{k}(G)$ consists of the polynomials whose image under the Chern-Weil homomorphism is an integral cohomology class. (See [7].)

ii) Note the following consequence of the geometric data 1.1. Let $U_{i}$ and $U_{j}$ be open subsets of $Z$ over which $E$ is trivial. Let $A_{i}(z)$ be the pull-back of the connection of the fibre $E_{z}$ to $\mathscr{P}$ via the trivialization

$$
U_{i} \times\left.\mathscr{P} \rightarrow E\right|_{U_{i}}
$$

and $A_{j}(z)$ correspondingly. If $g_{i j}$ denotes the transition function, then on $U_{i} \cap U_{j}$

$$
A_{j}(z)=A_{i}(z)^{g_{i j}(z)}
$$

is the gauge transformed connection of $A_{i}(z)$ by $g_{i j}(z)$.

Let $\mathscr{G}$ denote the group of gauge transformations of $\mathscr{P}$. If $\mathscr{G}$ is not connected it must be replaced by the connected component of the identity.

THEOREM 1.3. The geometric data 1.1 define a complex line bundle $\mathscr{L} \rightarrow Z$ with a Hermitian metric.

For the proof we need the following preparations: For the geometric data 1.1 and a set of trivializations $\varphi_{i}: U_{i} \times \mathscr{P} \rightarrow E \mid U_{i}$ with transition functions $g_{i j}$ as above we wish to construct transition functions $\theta_{i j}: U_{i} \cap U_{j} \rightarrow U$ (1) to get a line bundle. Let

$$
\tilde{g}_{i j}: U_{i} \cap U_{j} \times I \rightarrow \mathscr{G}
$$

be a homotopy from $g_{i j}$ to the identity such that $\tilde{g}_{i j}(z, 0)=\mathrm{id}$ and $\tilde{g}_{i j}(z, 1)=$ $g_{i j} \cdot \tilde{g}_{i j}$ can be considered as a gauge transformation in the bundle $\mathscr{P} \times I \rightarrow$ $X \times I$.

In general, for two connections $A_{0}$ and $A_{1}$ in a principal bundle $\mathbf{P} \rightarrow M$ over a manifold $M$, let $\mathbf{A}$ be the convex combination

$$
\mathbf{A}(p, s)=(1-s) A_{0}(p)+s A_{1}(p), \quad p \in \mathbf{P}, s \in[0,1]
$$

This is a connection in $\mathbf{P} \times I \rightarrow M \times I$. Let $P \in I_{0}^{k}(G)$ be an invariant polynomial of degree $k$ and let $F_{\mathbf{A}}$ be the curvature of the connection $\mathbf{A}$. Then $P\left(F_{\mathbf{A}}\right)$ is horizontal and the lift of a basic form, which will also be denoted by $P\left(F_{\mathbf{A}}\right)$. We define the differential $(2 k-1)$-form on $M$ :

DEFINITION 1.6.

$$
T P\left(A_{0}, A_{1}\right)=2 \pi \int_{s=0}^{1} P\left(F_{\mathbf{A}}\right) .
$$


Note that

$$
T P\left(A_{1}, A_{0}\right)=-T P\left(A_{0}, A_{1}\right) \text {. }
$$

In the following we apply this to the bundle $\mathscr{P} \times I \rightarrow X \times I$.

LEMMA 1.8. The form $T P\left(A_{i}, A_{i}^{\tilde{g}_{i j}}\right)$ is closed.

Proof. Let $i_{0}(x, t)=(x, t, 0)$ and $i_{1}(x, t)=(x, t, 1)$ be the inclusions $i_{v}$ : $X \times I \rightarrow X \times I \times I, v=0,1$, and let $A_{i j}(x, t, s)=(1-s) A_{i}(x)+s A_{i}(x)^{\tilde{g}_{i j}(x, t)}$. We have the equation ${ }^{\dagger}$

$$
d \int_{s=0}^{1} P\left(F_{A_{i j}}\right)-\int_{s=0}^{1} d P\left(F_{A_{i j}}\right)=i_{0}^{*} P\left(F_{A_{i j}}\right)-i_{1}^{*} P\left(F_{A_{i j}}\right) .
$$

$P$ is an invariant polynomial applied to a curvature form, so $P\left(F_{A_{i j}}\right)$ is closed. Hence

$$
\begin{aligned}
d T P\left(A_{i}, A_{i}^{\tilde{g}_{i j}}\right) & =i_{0}^{*} P\left(F_{A_{i j}}\right)-i_{1}^{*} P\left(F_{A_{i j}}\right) \\
& =P\left(F_{A_{i}}\right)-P\left(g_{i j}^{*} F_{A_{i}}\right) \\
& =P\left(F_{A_{i}}\right)-g_{i j}^{*} P\left(F_{A_{i}}\right) \\
& =0,
\end{aligned}
$$

since $P\left(F_{A_{i}}\right)$ is a basic form and the gauge transformation $g_{i j}$ acts as the identity on the base.

Define the function $\theta_{i j}$ on $U_{i} \cap U_{j}$ relative to a choice of a fixed connection $A_{0}$ in $\mathscr{P} \rightarrow X$.

DEFINITION 1.9.

$$
\theta_{i j}(z)=\exp \left(i \int_{X \times I} T P\left(A_{0}, A_{i}^{\tilde{g}_{i j}(z, t)}(z, x)\right)\right)
$$

In future calculations we shall omit the parameters $z, x$, and $t$.

Lemma 1.10. The function $\theta_{i j}$ is independent of the homotopy $\tilde{g}_{i j}$.

Proof. Let $\tilde{g}_{i j}^{1}$ and $\tilde{g}_{i j}^{2}$ be two homotopies of $g_{i j}$ to the identity and let $\theta_{i j}^{1}$

\footnotetext{
$\dagger$ The signs may look unusual but are in agreement with the sign convention of [5].
} 
and $\theta_{i j}^{2}$ be calculated by means of these two respectively. Then

$$
\begin{aligned}
\left(\theta_{i j}^{1}\right)^{-1} \theta_{i j}^{2} & =\exp i\left(-\int_{X \times I} T P\left(A_{0}, A_{i}^{\tilde{g}_{i j}^{1}}\right)+\int_{X \times I} T P\left(A_{0}, A_{i}^{\tilde{g}_{i j}^{2}}\right)\right) \\
& =\exp \left(i \int_{X \times S^{1}} T P\left(A_{0}, A_{i}^{g}\right)\right) \\
& =\exp \left(2 \pi i \int_{X \times S^{1}} \int_{s=0}^{1} P\left(F_{(1-s) A_{0}+s A_{i}^{g}}\right)\right) .
\end{aligned}
$$

Here, $g$ is the "homotopy" on $S^{1}$ made up of the two contributions $\tilde{g}_{i j}^{1}$ and $\tilde{g}_{i j}^{2}$. Then $g$ is a gauge transformation and hence an automorphism of the bundle $\mathscr{P} \times S^{1} \rightarrow X \times S^{1}$. Indeed,

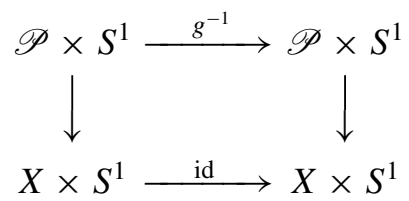

commutes. Now consider the mapping torus of $\mathscr{P} \times S^{1}$ given by

$$
T=\left(\mathscr{P} \times S^{1}\right) \times I / \sim
$$

where we identify $((p, t), 0) \sim\left(g^{-1} \cdot(p, t), 1\right) . T$ is diffeomorphic to $\mathscr{P} \times$ $S^{1} \times S^{1}$. The convex combination $(1-s) A_{0}+s A_{i}^{g}$ is a connection in the bundle $\mathscr{P} \times S^{1} \times I$, but by the construction of the mapping torus, it becomes a connection in $T$. In fact, the connection in $\mathscr{P} \times S^{1} \times I$ at $((p, t), 0)$ is $A_{0}$, and the connection at $\left(g^{-1} \cdot(p, t), 1\right)$ is $A_{i}^{g}$. The connection at $((p, t), 0)$ should equal the pull-back along the identification map of the connection at $\left(g^{-1} \cdot(p, t), 1\right)$. But the pull-back of $A_{0}^{g}$ along $g^{-1}$. is just $\left(g^{-1}\right)^{*} A_{0}^{g}=A_{0}^{g g^{-1}}=A_{0}$. With this in mind we can make a replacement of the integral from above: If we let $\tilde{A}$ be the connection on $T$ obtained from $(1-s) A_{0}+s A_{i}^{g}$ under the mapping torus construction,

$$
\int_{X \times S^{1}} \int_{s=0}^{1} P\left(F_{(1-s) A_{0}+s A_{i}^{g}}\right)=\int_{T} P\left(F_{\tilde{A}}\right) .
$$

This is the integral of an invariant polynomial $P \in I_{0}^{k}(G)$ over a closed manifold, and the result is an integer. Multiplying by $2 \pi i$ and taking exp concludes the proof.

LEMMA 1.12. Let $U_{i}, U_{j}$ and $U_{k}$ be three open subsets of $Z$ with nonempty intersection. Then $\theta_{i j}, \theta_{j k}$ and $\theta_{i k}$ satisfy the cocycle condition, $\theta_{i j} \theta_{j k}=\theta_{i k}$. 
Proof. For $z \in U_{i} \cap U_{j} \cap U_{k}$,

$$
\theta_{i j} \theta_{j k}=\exp i\left(\int_{X \times I} T P\left(A_{0}, A_{i}^{\tilde{g}_{i j}}\right)+\int_{X \times I} T P\left(A_{0}, A_{j}^{\tilde{g}_{j k}}\right)\right) .
$$

Since $A_{i}^{\tilde{g}_{i j}(z, 1)}=A_{j}$ and $A_{j}^{\tilde{g}_{j k}(z, 0)}=A_{j}$, the integrands agree at the endpoints of the intervals, and after a slight reparametrization,

$$
\tilde{g}_{i k}(z, t)= \begin{cases}\tilde{g}_{i j}(z, 2 t) & \text { if } 0 \leq t \leq \frac{1}{2} \\ g_{i j} \tilde{g}_{j k}(z, 2 t-1) & \text { if } \frac{1}{2} \leq t \leq 1\end{cases}
$$

is a homotopy from the identity to $g_{j k}$. Hence the sum of the two integrals above is

$$
\int_{X \times I} T P\left(A_{0}, A_{i}^{\tilde{g}_{i k}}\right)
$$

and the result follows by lemma 1.8 .

Proof of Theorem 1.3. The functions in definition 1.9 are well-defined by lemma 1.10 and satisfy the cocycle condition by lemma 1.12 . Hence the $\left\{\theta_{i j}\right\}$ 's are transition functions of a line bundle $\mathscr{L}$. Note that the transition functions are $U(1)$-valued, and hence $\mathscr{L}$ can be equipped with a Hermitian metric. This concludes the proof of theorem 1.3.

Proposition 1.13. The isomorphism class of $\mathscr{L}$ is independent of the choice of $A_{0}$.

Proof. For the proof of this we need to rewrite the transition functions $\theta_{i j}$. Let $\Delta^{2}$ be the two-simplex

$$
\Delta^{2}=\left\{s_{0}, s_{1}, s_{2} \in \mathrm{R} \mid s_{0}+s_{1}+s_{2}=1, s_{i} \geq 0 \text { for } i=0,1,2\right\} .
$$

For three connections $A_{0}, A_{1}$, and $A_{2}$ of the principal bundle $\mathscr{P} \rightarrow X$ the set of convex combinations $\left\{s_{0} A_{0}+s_{1} A_{1}+s_{2} A_{2} \mid\left(s_{0}, s_{1}, s_{2}\right) \in \Delta^{2}\right\}$ will be a connection of the bundle $\mathscr{P} \times \Delta^{2} \rightarrow X \times \Delta^{2}$. Define the differential $(2 k-2)$-form on $X^{\dagger}$

$$
T P\left(A_{0}, A_{1}, A_{2}\right)=2 \pi \int_{\Delta^{2}} P\left(F_{s_{0} A_{0}+s_{1} A_{1}+s_{2} A_{2}}\right) .
$$

An easy calculation shows that

$$
d T P\left(A_{0}, A_{1}, A_{2}\right)=-\sum_{i=0}^{2}(-1)^{i} T P\left(\ldots, \hat{A}_{i}, \ldots\right),
$$

\footnotetext{
${ }^{\dagger}$ The form $P\left(F_{S_{0} A_{0}+s_{1} A_{1}+s_{2} A_{2}}\right)$ is horizontal and can be identified with a basic form.
} 
where $T P\left(\ldots, \hat{A}_{i}, \ldots\right)$ is the $(2 k-1)$-form from definition 1.6 with $A_{i}$ omitted. Inserting the three connections $A_{0}, A_{i}$ and $A_{i}^{\tilde{g}_{i j}}$ into $d T P$ and integrating over $X \times I$ we get

$$
\int_{X \times I} d T P\left(A_{0}, A_{i}, A_{i}^{\tilde{g}_{i j}}\right)=\int_{X \times I} T P\left(A_{0}, A_{i}^{\tilde{g}_{i j}}\right)-\int_{X \times I} T P\left(A_{i}, A_{i}^{\tilde{g}_{i j}}\right),
$$

since $\int_{X \times I} T P\left(A_{0}, A_{i}\right)=0$ from a dimension argument. On the other hand,

$$
\int_{X \times I} d T P\left(A_{0}, A_{i}, A_{i}^{\tilde{g}_{i j}}\right)=\int_{X} T P\left(A_{0}, A_{i}, A_{j}\right),
$$

and it follows that

$$
\int_{X \times I} T P\left(A_{0}, A_{i}^{\tilde{g}_{i j}}\right)=\int_{X \times I} T P\left(A_{i}, A_{i}^{\tilde{g}_{i j}}\right)+\int_{X} T P\left(A_{0}, A_{i}, A_{j}\right) .
$$

Let $\Delta^{3}$ denote the three-simplex and for four connections $A_{0}, A_{1}, A_{2}, A_{3}$ define the $(2 k-3)$-form on $X$

$$
T P\left(A_{0}, A_{1}, A_{2}, A_{3}\right)=2 \pi \int_{\Delta^{3}} P\left(F_{S_{0} A_{0}+s_{1} A_{1}+s_{2} A_{2}+s_{3} A_{3}}\right)
$$

as above, but in one dimension higher. We have

$$
d T P\left(A_{0}, A_{1}, A_{2}, A_{3}\right)=-\sum_{i=0}^{3}(-1)^{i} T P\left(\ldots, \hat{A}_{i}, \ldots\right),
$$

where the $T P$ 's in the sum are the $(2 k-2)$-forms from above. Also note that $\int_{X} d T P\left(A_{0}, A_{1}, A_{2}, A_{3}\right)=0$. Now let $A_{0}$ and $A_{1}$ be two fixed connections of $\mathscr{P}$, and for $v=0,1$ let

$$
\theta_{i j}^{v}=\exp i \int_{X \times I} T P\left(A_{0}, A_{i}^{\tilde{g}_{i j}}\right) .
$$

Rewriting the integrals as in (1.15) and applying (1.16) yields

$$
\begin{aligned}
\theta_{i j}^{0}\left(\theta_{i j}^{1}\right)^{-1} & =\exp i\left(\int_{X} T P\left(A_{0}, A_{i}, A_{j}\right)-\int_{X} T P\left(A_{1}, A_{i}, A_{j}\right)\right) \\
& =\exp i\left(\int_{X} T P\left(A_{0}, A_{1}, A_{i}\right)-\int_{X} T P\left(A_{0}, A_{1}, A_{j}\right)\right),
\end{aligned}
$$

but this is a coboundary and hence the $\left\{\theta_{i j}^{0}\right\}$ 's and the $\left\{\theta_{i j}^{1}\right\}$ 's define isomorphic line bundles. 
REMARK 1.17. Note that the proof provides explicit isomorphisms.

Proposition 1.18. Let $X$ be a closed surface, let $\mathscr{P}=X \times G$ be the product bundle and $P=-C_{2}=-\frac{1}{8 \pi^{2}} \operatorname{Tr}$, minus the second Chern polynomial. Then the transition functions $\theta_{i j}$ define the same line bundle as the one of Ramadas, Singer and Weitsman in [14].

Proof. First recall that the line bundle of Ramadas, Singer and Weitsman is constructed by means of a 3-manifold $Y$ that has $X$ as boundary and by defining the Chern-Simons functional

$$
C S(\bar{A})=\frac{1}{4 \pi} \int_{Y} \operatorname{Tr}\left(\iota^{*}\left(\bar{A} d \bar{A}-\frac{2}{3} \bar{A} \bar{A} \bar{A}\right)\right) \quad \bmod 2 \pi \mathrm{Z} .
$$

Then a cocycle is defined on $\mathscr{A} \times \mathscr{G}$, where $\mathscr{A}$ is the space of connections of $X \times S U(2)$ and $\mathscr{G}$ is the group of gauge transformations, by

$$
\Theta(A, g)=\exp i\left(C S\left(\bar{A}^{\bar{g}}\right)-C S(\bar{A})\right)
$$

where $\bar{A}$ is an extension of $A$ into $Y$ and $\bar{g}$ is an extension of $g$. This gives a line bundle on the manifold $\mathscr{A}_{F}^{s} / \mathscr{G}$, the set of flat, irreducible connections of $X \times S U(2)$ modulo $\mathscr{G}$. Given a covering $\left\{U_{i}\right\}$ and transition functions $\left\{g_{i j}\right\}$, the transition functions in the line bundle are given by $\Theta\left(A_{i}, g_{i j}\right)$ and we shall show that this equals $\theta_{i j}$.

Recalling that the curvature of the connection $A$ is given by $F_{A}=A \wedge A+d A$ we get

$$
\begin{aligned}
T P\left(A_{0}, A_{i}^{\tilde{g}_{i j}}\right) & =-\frac{i}{4 \pi} \int_{s=0}^{1} \operatorname{Tr}\left(F_{s A_{0}+(1-s) A_{i}^{\tilde{g}_{i j}}}^{2}\right) \\
& =-\frac{i}{4 \pi} \int_{s=0}^{1} \operatorname{Tr}\left(\left(s A_{0}+(1-s) A_{i}^{\tilde{g}_{i j}}\right)^{2}+d\left(s A_{0}+(1-s) A_{i}^{\tilde{g}_{i j}}\right)\right)^{2}
\end{aligned}
$$

Calculation of this integral yields

$$
-\left(A_{i}^{\tilde{g}_{i j}} \wedge d A_{i}^{\tilde{g}_{i j}}+\frac{2}{3}\left(A_{i}^{\tilde{g}_{i j}}\right)^{3}\right)+\left(A_{0} \wedge d A_{0}+\frac{2}{3} A_{0}^{3}\right)-d\left(A_{0} \wedge A_{i}^{\tilde{g}_{i j}}\right),
$$

and when integrated over $X \times I$ the terms in parentheses vanish, as does the term $d\left(A_{0} \wedge A_{i}^{\tilde{g}_{i j}}\right)$. In fact we get the terms $A_{0} \wedge A_{i}$ and $A_{0} \wedge A_{j}$ with opposite signs from the two ends of the cylinder, and since $A_{i}$ and $A_{j}$ are gauge equivalent the two terms cancel out each other when pulled back to the base. What is left is then

$$
\theta_{i j}=\exp \frac{1}{4 \pi} \int_{X \times I} \operatorname{Tr}\left(A_{i}^{\tilde{g}_{i j}} \wedge d A_{i}^{\tilde{g}_{i j}}+\frac{2}{3}\left(A_{i}^{\tilde{g}_{i j}}\right)^{3}\right) .
$$


Now let $W$ be the closed manifold $Y \cup(X \times I) \cup(-Y)$, where $-Y$ denotes $Y$ with the opposite orientation. The integrands of (1.19) and (1.20) agree at the boundaries of the constituents of $W$, and letting $B$ denote the connection

$$
B= \begin{cases}\bar{A}_{i} & \text { on } Y \\ A_{i}^{\tilde{g}_{i j}} & \text { on } X \times I \\ \bar{A}_{j} & \text { on }-Y\end{cases}
$$

we have

$$
\Theta\left(A_{i}, g_{i j}\right)^{-1} \theta_{i j}=\exp i\left(\frac{1}{4 \pi} \int_{W} \operatorname{Tr}\left(\iota^{*}\left(B d B+\frac{2}{3} B^{3}\right)\right)\right) .
$$

This contains an integral of a Chern-Simons form over a closed manifold. Hence the contents of the parentheses is an integer multiple of $2 \pi$, and the whole expression equals 1 , which completes the proof.

In [14], Ramadas, Singer, and Weitsman show that the line bundle $\mathscr{L}$ defined by the transition functions in definition 1.9 is isomorphic to the Quillen determinant line bundle $\mathscr{L}_{D}$ that arises from the family $\left\{\bar{\partial}_{A} \mid A \in \mathscr{A}_{F}^{s}\right\}$.

REMARK 1.21. A slightly more general version of the line bundle is obtained if we consider two fibre bundles $E$ and $F$ as in the geometric data 1.1 with families of connections $A_{z}$ and $B_{z}$ respectively, both with local trivializations as in (1.2) and with homotopies $\tilde{g}_{i j}^{A}$ and $\tilde{g}_{i j}^{B}$ respectively as in (1.4). Define transition functions $\theta_{i j}^{A B}=\exp i \int_{X \times I} T P\left(B_{i}^{\tilde{g}_{i j}^{B}}, A_{i}^{\tilde{g}_{i j}^{A}}\right)$. It is an easy calculation to show that $\theta_{i j}^{A B}$ differ from the product $\theta_{i j}^{A}\left(\theta_{i j}^{B}\right)^{-1}$ by a coboundary and hence define the same line bundle. Here $\theta_{i j}^{A}$ and $\theta_{i j}^{B}$ denote the transition functions of the $A$-family and the $B$-family respectively. In other words, given the two families $A$ and $B$ we get a line bundle $\mathscr{L}^{A B}$. If the $B$-family is constant and equal to $A_{0}$ we get the same line bundle as the one defined by the transition functions in definition 1.9. If $H$ is a third fibre bundle with connections $C$ we get three relative line bundles, $\mathscr{L}^{A B}, \mathscr{L}^{B C}$, and $\mathscr{L}^{A C}$, and it is not difficult to show that there is an isomorphism $\mathscr{L}^{A B} \otimes \mathscr{L}^{B C} \cong \mathscr{L}^{A C}$.

\section{Connections in the Line Bundle}

In this section we shall describe connections of the line bundle $\mathscr{L}$ constructed in the previous section.

THEOREM 2.1. Given the geometric data 1.1, let A be a connection of the principal bundle $E \rightarrow Z \times X$ which fibre-wise restricts to the connection of each fibre of the bundle $E \rightarrow Z$. Then the induced line bundle $\mathscr{L}$ with transition 
functions $\theta_{i j}$ from definition 1.9 has a canonical Hermitian connection whose curvature is

$$
2 \pi i \int_{X} P\left(F_{A}\right)
$$

REMARK 2.2. Thus, for a connection in $\mathscr{L}$ we need an extension of the family $\{A(z)\}$ to a connection also in the $Z$ direction. However, corollary 2.10 below gives conditions (e.g. when $A(z)$ is flat for all $z \in Z$ ) insuring the connection to be independent of choice of extension. Notice also that such an extension always exists. This is easily seen by considering the fibre bundle $E$ as a principal $G$-bundle, which locally, has the form $G \rightarrow U_{i} \times \mathscr{P} \rightarrow U_{i} \times X$. Pulling back $A_{i}(z)$ via $U_{i} \times \mathscr{P} \rightarrow \mathscr{P}$ and using partition of unity yields a connection of $E \rightarrow Z \times X$.

In the following we shall see how a connection in $E \rightarrow Z \times X$ gives rise to a canonical connection of the line bundle $\mathscr{L}$. Again choose a fixed connection $A_{0}$ in $\mathscr{P} \rightarrow X$, and let $\theta_{i j}$ be the transition functions for $\mathscr{L}$ given by definition 1.9. The local connection one-forms are now given as follows.

Definition 2.3. Let $\omega_{i}$ be the 1 -form on $U_{i} \subseteq Z$

$$
\omega_{i}=-i \int_{X} T P\left(A_{0}, A_{i}\right)
$$

LEMMA 2.4.

$$
\theta_{i j}^{-1} d \theta_{i j}=i\left(-\int_{X} T P\left(A_{0}, A_{i}\right)+\int_{X} T P\left(A_{0}, A_{j}\right)\right) .
$$

Proof. By (1.15),

$$
\theta_{i j}=\exp i\left(\int_{X \times I} T P\left(A_{i}, A_{i}^{\tilde{g}_{i j}}\right)+\int_{X} T P\left(A_{0}, A_{i}, A_{j}\right)\right),
$$

where $A_{i}$ and $A_{j}$ are the pull-backs of the connections in $E_{\mid U_{i}} \rightarrow U_{i}$ and $E_{\mid U_{j}} \rightarrow U_{j}$. It is obvious that

$$
\begin{aligned}
d \theta_{i j} & =d \exp i\left(\int_{X \times I} T P\left(A_{i}, A_{i}^{\tilde{g}_{i j}}\right)+\int_{X} T P\left(A_{0}, A_{i}, A_{j}\right)\right) \\
& =i \theta_{i j} d\left(\int_{X \times I} T P\left(A_{i}, A_{i}^{\tilde{g}_{i j}}\right)+\int_{X} T P\left(A_{0}, A_{i}, A_{j}\right)\right)
\end{aligned}
$$


and so

$$
\theta_{i j}^{-1} d \theta_{i j}=i\left(d \int_{X \times I} T P\left(A_{i}, A_{i}^{\tilde{g}_{i j}}\right)+d \int_{X} T P\left(A_{0}, A_{i}, A_{j}\right)\right) .
$$

Note that this is a differential form on $U_{i} \cap U_{j}$ and of course depends on $z \in U_{i} \cap U_{j}$. The two terms are treated separately. First,

$$
d_{Z} \int_{X \times I} T P\left(A_{i}, A_{i}^{\tilde{g}_{i j}}\right)=\int_{X \times I} d T P\left(A_{i}, A_{i}^{\tilde{g}_{i j}}\right)-\int_{X \times I} d_{X \times I} T P\left(A_{i}, A_{i}^{\tilde{g}_{i j}}\right) .
$$

The integral $\int_{X \times I} d T P\left(A_{i}, A_{i}^{\tilde{g}_{i j}}\right)$ is zero by lemma 1.8 . The second term evaluates to

$$
\int_{X \times I} d_{X \times I} T P\left(A_{i}, A_{i}^{\tilde{g}_{i j}}\right)=-\int_{X} T P\left(A_{i}, A_{j}\right)
$$

The second integral in (2.5) is treated in the same way, i.e.

$$
d_{Z} \int_{X} T P\left(A_{0}, A_{i}, A_{j}\right)=\int_{X} d T P\left(A_{0}, A_{i}, A_{j}\right)-\int_{X} d_{X} T P\left(A_{0}, A_{i}, A_{j}\right) .
$$

Stokes' theorem shows that the second term in this expression is zero, and by (1.14),

$$
\int_{X} d T P\left(A_{0}, A_{i}, A_{j}\right)=\int_{X}\left(-T P\left(A_{0}, A_{i}\right)+T P\left(A_{0}, A_{j}\right)-T P\left(A_{i}, A_{j}\right)\right) .
$$

This leads to

$$
\theta_{i j}^{-1} d \theta_{i j}=i\left(-\int_{X} T P\left(A_{0}, A_{i}\right)+\int_{X} T P\left(A_{0}, A_{j}\right)\right) .
$$

Note that $\omega_{i}$ is purely imaginary, and hence the connection is Hermitian.

Next, we calculate the curvature of $\omega_{i}$. Since $\mathscr{L}$ is a line bundle, $\omega \wedge \omega=0$, and the curvature of $\omega$ is just $d \omega$. It suffices to show that

$$
d \omega_{i}=2 \pi i \int_{X} P\left(F_{A_{i}}\right)
$$

This is a direct calculation. According to the sign convention, integration along 
the fibre commutes with the differential $d_{Z}$.

$$
\begin{aligned}
d \omega_{i} & =-i d \int_{X} T P\left(A_{0}, A_{i}\right) \\
& =-2 \pi i d_{Z} \int_{X} \int_{s=0}^{1} P\left(F_{(1-s) A_{0}+s A_{i}}\right) \\
& =-2 \pi i \int_{X} \int_{s=0}^{1} d_{Z} P\left(F_{\left.(1-s) A_{0}+s A_{i}\right)}\right) \\
& =-2 \pi i \int_{X} \int_{s=0}^{1}\left(d-d_{X}-d_{s}\right) P\left(F_{(1-s) A_{0}+s A_{i}}\right) \\
& =-2 \pi i \int_{X} \int_{s=0}^{1} d P\left(F_{(1-s) A_{0}+s A_{i}}\right)+2 \pi i \int_{X} d_{X} \int_{s=0}^{1} P\left(F_{(1-s) A_{0}+s A_{i}}\right) \\
& \quad+2 \pi i \int_{X} \int_{s=0}^{1} d_{S} P\left(F_{(1-s) A_{0}+s A_{i}}\right) \\
= & 2 \pi i \int_{X} P\left(F_{A_{i}}\right)-2 \pi i \int_{X} P\left(F_{A_{0}}\right) \\
= & 2 \pi i \int_{X} P\left(F_{A_{i}}\right),
\end{aligned}
$$

since the terms containing $d$ and $d_{X}$ vanish; the form $2 \pi i \int_{X} P\left(F_{A_{0}}\right)$ vanishes, since it is independent of $z \in Z$.

This concludes the proof of theorem 2.1.

We shall now investigate how the connection of the line bundle depends on the connection $A$ of the principal bundle $E \rightarrow Z \times X$. Given a connection $A^{E}$ in $E, A^{E}$ can be written locally as

$$
A_{i}^{E}=A_{i}+B_{i}
$$

where $A_{i}$ contains all terms involving derivations in $\mathscr{P}$-direction ( $d p$ 's) and $B_{i}$ contains all terms involving derivations in $Z$-direction $(d z$ 's). If two different connections $A_{1}^{E}$ and $A_{2}^{E}$ in $E$ induce the same family $\left\{A_{i}(z)\right\}_{z \in U_{i}}$ in $\mathscr{P}$ then, locally

$$
A_{1, i}^{E}=A_{i}+B_{1, i}, \quad A_{2, i}^{E}=A_{i}+B_{2, i}
$$

because both $A_{1, i}^{E}$ and $A_{2, i}^{E}$ restrict to $A_{i}(z)$ for fixed $z$.

THEOREM 2.8. Let $A_{1}$ and $A_{2}$ be two connections of the bundle $E \rightarrow Z \times X$. Let $\omega_{1}$ and $\omega_{2}$ be two connections in the associated line bundle as defined in 
definition 2.3. Assume that both $A_{1}$ and $A_{2}$ restrict to $A(z)$ for each $z \in Z$. Then

$$
\omega_{2}-\omega_{1}=-k \int_{X} P\left(F_{A}^{k-1} \wedge \beta\right) .
$$

Here $k$ is the degree of $P$ and $\beta=A_{2}-A_{1}$ is a horizontal 1-form in $E \rightarrow Z$ so that the integral only involves the curvatures $F_{A(z)}$ along the fibres.

Proof. Consider a subset $U_{i} \subseteq Z$ such that the local considerations from above apply, i.e. $\omega_{i}$ can be calculated explicitly by the expression in definition 2.3. In the proof the index $i$ is left out. The first step is to show that $\omega_{2}-\omega_{1}=-i \int_{X} T P\left(A_{1}, A_{2}\right)$. It has already been shown that

$$
\begin{aligned}
d_{Z} \int_{X} T P\left(A_{0}, A_{1}, A_{2}\right) & \\
& =-\int_{X} T P\left(A_{0}, A_{1}\right)+\int_{X} T P\left(A_{0}, A_{2}\right)-\int_{X} T P\left(A_{1}, A_{2}\right) .
\end{aligned}
$$

Hence it suffices to show that $d_{Z} \int_{X} T P\left(A_{0}, A_{1}, A_{2}\right)=0$. Write $A_{1}=A+B_{1}$ and $A_{2}=A+B_{2}$ and consider

$$
\int_{X} T P\left(A_{0}, A_{1}, A_{2}\right)=2 \pi \int_{X} \int_{\Delta^{2}} P\left(F_{S_{0} A_{0}+s_{1} A_{1}+s_{2} A_{2}}\right)
$$

which is a function on $Z$ and therefore can be calculated pointwise. Let $\mathbf{A}=$ $s_{0} A_{0}+s_{1} A_{1}+s_{2} A_{2}$ and write $F_{\mathbf{A}}=d \mathbf{A}+\mathbf{A} \wedge \mathbf{A}$ as

$$
F_{\mathbf{A}}=\sigma+\phi+\psi,
$$

where $\sigma$ contains all terms involving $d s$ 's, $\phi$ contains all terms involving $d x$ 's only, and $\psi$ contains all terms involving $d z$ 's. Now, a term in the integral

$$
\int_{X} \int_{\Delta^{2}} P\left(F_{\mathbf{A}} \wedge \ldots \wedge F_{\mathbf{A}}\right)
$$

contributes with something non-vanishing when it contains exactly $(2 k-2)$ $d x$ 's and two $d s$ 's, i.e. such terms contain no $\psi$ 's. Hence, one can replace both $A_{1}$ and $A_{2}$ by $A$ and calculate:

$$
d_{Z} \int_{X} T P\left(A_{0}, A_{1}, A_{2}\right)=d_{Z} \int_{X} T P\left(A_{0}, A, A\right)=0 .
$$

This concludes the first step.

The second step deals with $\int_{X} T P\left(A_{1}, A_{2}\right)$. Recall that $T P\left(A_{1}, A_{2}\right)$ is given by $2 \pi \int_{s=0}^{1} P\left(F_{(1-s) A_{1}+s A_{2}}\right)$. Splitting up $A_{1}$ and $A_{2}$ as in (2.7) gives

$$
(1-s) A_{1}+s A_{2}=A+B_{1}+s\left(B_{2}-B_{1}\right) \text {. }
$$


Then a calculation yields

$$
F_{(1-s) A_{1}+s A_{2}}=F_{A}+d s \wedge \beta+\gamma,
$$

where $\beta=B_{2}-B_{1}$ and $\gamma$ do not contain any terms involving $d s$ or $d x$ 's. Since $P$ is an invariant polynomial of degree $k$,

$$
\begin{aligned}
\int_{s=0}^{1} P\left(F_{(1-s) A_{1}+s A_{2}}\right)= & \int_{s=0}^{1} P\left(F_{(1-s) A_{1}+s A_{2}} \wedge \ldots \wedge F_{(1-s) A_{1}+s A_{2}}\right) \\
= & \int_{s=0}^{1} P\left(\left(F_{A}+\beta+\gamma\right) \wedge \ldots \wedge\left(F_{A}+\beta+\gamma\right)\right) \\
= & \int_{s=0}^{1} P\left(F_{A}^{k-1} \wedge \beta+F_{A}^{k-2} \wedge \beta \wedge F_{A}+\cdots+\beta \wedge F_{A}^{k-1}\right) \\
& \quad+\int_{s=0}^{1} R(s, x, z) \\
= & k \int_{s=0}^{1} P\left(F_{A}^{k-1} \wedge \beta\right)+\int_{s=0}^{1} R(s, x, z) \\
= & -k P\left(F_{A}^{k-1} \wedge \beta\right)+\int_{s=0}^{1} R(s, x, z),
\end{aligned}
$$

where $R$ contains all terms which are not on the form $F_{A}^{k-1} \wedge \beta$. Note that all the terms containing $2 k-2$ derivations in the $X$-direction have been accounted for since such forms must contain $(k-1)$ times $F_{A}$. The form $R(s, x, z)$ contains no such terms and hence

$$
\int_{X} \int_{s=0}^{1} R(s, x, z)=0 .
$$

This concludes the proof.

Corollary 2.10. Assume that $A_{1}=A+B_{1}$ and $A_{2}=A+B_{2}$ are two connections in $E$ which agree along the fibres of $E \rightarrow Z$. If $F_{A_{z}}^{k-1}=0$ for each $z$, then $A_{1}$ and $A_{2}$ induce the same connection $\omega_{i}$ in the associated line bundle. Furthermore, if $k \geq 3$ and $F_{A_{z}}^{k-2}=0$ for each $z$, then $\mathscr{L}$ is a flat bundle. In particular if $A_{z}$ is flat for each $z$, then the connection in $\mathscr{L}$ is well-defined for $k \geq 2$ and is flat for $k \geq 3$.

Proof. This follows immediately from theorem 2.8 and theorem 2.1.

REMARK 2.11. This construction also gives a tensor power of the line bundle constructed by Freed and Bismut (see [10]) for a family of Riemannian manifolds at least in the case of varying metrics on a fixed manifold $X$. In this case 
let $\mathscr{P}_{z}=F(X)$ be the oriented orthogonal frame bundle of $X$ with the LeviCivita connection $A_{z}$ of the metric of the fibre, and let $P$ be the $\hat{A}$-polynomial multiplied by the least common denominator $\mu$ of its coefficients (see [12], Chapter I, $\S 1.6)$. The curvature of the line bundle is $2 \pi i \int_{X} \mu \hat{A}\left(F_{A}\right)$.

EXAMPLE 2.12. The case of [14]. Let $X$ be a closed surface and $\mathscr{P}=X \times$ $S U$ (2). To compare our connection and its curvature to the case in [14] consider $\omega$ as a 1 -form on $\mathscr{A}_{F}$. Let $A \in \mathscr{A}_{F}$, and $\alpha \in T_{A} \mathscr{A}_{F}$. Let $\gamma:(-\epsilon, \epsilon) \rightarrow \mathscr{A}$ be a curve such that $\gamma(0)=A$, and $\gamma^{\prime}(0)=\alpha$. Then

$$
\omega(\alpha)=\omega\left(\gamma_{*}\left(\frac{d}{d t}\right)\right)=\gamma^{*} \omega\left(\frac{d}{d t}\right) .
$$

Since $\mathscr{A}$ is an affine space we can let $\gamma(t)=A+t \alpha$. We wish to compare $\omega$ to the form $\hat{\omega}_{\mathrm{RSW}}$ from [14] given by $\hat{\omega}_{\mathrm{RSW}}(\alpha)=\frac{i}{4 \pi} \int_{X} \operatorname{Tr}(A \wedge \alpha)$. Write $A$ as $A=A_{0}+B$, where $A_{0}$ is a flat connection. Then

(1) $T P\left(A_{0}, A\right)=\frac{1}{4 \pi} \operatorname{Tr}\left(B d_{A_{0}} B+\frac{2}{3} B^{3}\right)$

(2) $\omega(\alpha)=\frac{i}{4 \pi} \int_{X} \operatorname{Tr}(B \wedge \alpha)$,

where in this case $P=-C_{2}=-\frac{1}{8 \pi^{2}}$ Tr is minus the second Chern polynomial. The curvature is obtained from this and yields

$$
d \omega(\alpha, \beta)=\frac{i}{2 \pi} \int_{X} \operatorname{Tr}(\alpha \wedge \beta)
$$

in agreement with [14].

REMARK 2.13. The connection one-form of the relative line bundle described in Remark (1.27) is given by $\omega_{i}^{A B}=-i \int_{X} T P\left(B_{i}, A_{i}\right)$.

\section{The Chern-Simons Invariant}

We shall now extend the definition of the Chern-Simons invariant to a family of bundles and connections over a family of odd-dimensional manifolds with boundary. In this situation the Chern-Simons invariant determines a section of the inverse line bundle $\mathscr{L}^{-1}$, where $\mathscr{L}$ is the line bundle constructed in section 1 for the family of boundaries. In the case of a single bundle $\overline{\mathscr{P}}$ with connection $\bar{A}$ over an odd-dimensional manifold $Y$ with boundary $X$ the Chern-Simons invariant of $\bar{A}$ must be defined relative to some "boundary conditions". For these we take once and for all a fixed manifold $Y_{0}$ with $\partial Y_{0}=X$ together with a principal bundle $\overline{\mathscr{P}}_{0} \rightarrow Y_{0}$ with connection $\bar{A}_{00}$ extending our background connection $A_{0}$ on $\mathscr{P}$ over $X$. In the special case of $\mathscr{P}=X \times G$ we can take $\overline{\mathscr{P}}_{0}=Y_{0} \times G$ and $A_{00}$ the Maurer-Cartan connection. With these data we can now define the relative Chern-Simons invariant $\operatorname{cs}\left(\bar{A}, \bar{A}_{00}\right)$ for $P \in I_{0}^{*}(G)$ 
as follows. Consider the "glued" manifold $W=Y \cup(X \times I) \cup\left(-Y_{0}\right)$ with $G$-bundle $\overline{\mathscr{P}} \cup(X \times I \times G) \cup \overline{\mathscr{P}}_{0}$ and connection $\bar{B}$ given by

$$
\bar{B}= \begin{cases}\bar{A} & \text { on } Y \\ (1-t) A+t A_{0} & \text { on } X \times I \times G \\ \bar{A}_{00} & \text { on }-Y_{0}\end{cases}
$$

Then we put

$$
\operatorname{cs}\left(\bar{A}, \bar{A}_{00}\right)=\exp \left(2 \pi i\left\langle S_{P}(\bar{B}),[W]\right\rangle\right),
$$

where $S_{P}(\bar{B})$ is the secondary characteristic class for the connection $\bar{B}$ as defined by Cheeger-Chern-Simons [7] or [6] (see also [9]).

Returning to the case of a family we thus have the following general setup with the above "boundary conditions" as point (5):

Geometric Data 3.2.

(1)-(4) as in the geometric data 1.1

(5) A smooth, compact, oriented, odd-dimensional manifold $Y_{0}$ with $\partial Y_{0}=X$ and a principal $G$-bundle $\overline{\mathscr{P}}_{0} \rightarrow Y_{0}$ which extends $\mathscr{P}$, i.e. $\left.\overline{\mathscr{P}}_{0}\right|_{X}=\mathscr{P}$, and a connection $\bar{A}_{00}$ which extends $A_{0}$.

(6) A smooth, compact, oriented, odd-dimensional manifold $Y$ with $\partial Y=$ $X$ and a principal $G$-bundle $\overline{\mathscr{P}} \rightarrow Y$ which extends $\mathscr{P}$, i.e. $\left.\overline{\mathscr{P}}\right|_{X}=$ $\mathscr{P}$,

(7) A fibre bundle $\bar{P} \rightarrow \bar{E} \rightarrow Z$, where each fibre has a connection $\bar{A}_{z}$ which extends $A_{z}$ and such that the transition functions are gauge transformations homotopic to the identity

THEOREM 3.3. The geometric data 3.2 determine a global section cs of the inverse line bundle $\mathscr{L}^{-1} \rightarrow Z$. Furthermore, for an extension of $\bar{A}$ to a connection in the G-bundle $\bar{E} \rightarrow Z \times Y$, the covariant derivative of cs with respect to the connection of section 2 is

$$
\nabla_{\bar{A}}(c s)=2 \pi i \int_{Y} P\left(F_{\bar{A}}\right) \otimes c s .
$$

COROLlary 3.4. Given the geometric data 3.2 the associated line bundle $\mathscr{L}$ is trivial.

For the proof of theorem 3.3 we choose a covering $\left\{U_{i}\right\}$ of $Z$ and local trivializations of $\bar{E}$

$$
\bar{\varphi}_{i}: \bar{E}_{\mid U_{i}} \rightarrow U_{i} \times \overline{\mathscr{P}} .
$$


This gives rise to a connection $\bar{A}_{i}(z)=\left(\bar{\varphi}_{i}^{-1}\right)^{*} \bar{A}_{z}$ in $\overline{\mathscr{P}}$ for each $z \in Z$. The trivializations give transition functions over $U_{i^{\prime}} \cap U_{j^{\prime}}$ :

$$
\bar{g}_{i j}: U_{i} \cap U_{j} \rightarrow \overline{\mathscr{G}},
$$

where $\overline{\mathscr{G}}$ is the group of gauge transformations of $\overline{\mathscr{P}}$. Of course these trivializations restrict to local trivializations of the boundary. Also choose a connection $\bar{A}_{0}$ in $\bar{P} \rightarrow Y$ extending $A_{0}$.

Define a section of the inverse line bundle $\mathscr{L}^{-1}=\mathscr{L}^{*}$ as follows. Over $U_{i}$, the section is defined by

$$
c s_{i}(z)=\operatorname{cs}\left(\bar{A}_{0}, \bar{A}_{00}\right) \cdot \exp i\left(-\int_{Y} T P\left(\bar{A}_{0}, \bar{A}_{i}\right)\right) .
$$

Lemma 3.8. The local sections defined in (3.7) patch together to a global section of $\mathscr{L}^{*}$, independent of choice of $\bar{A}_{0}$.

Proof. On $U_{i} \cap U_{j}$ the transition function $\theta_{i j}$ "from $U_{i}$ to $U_{j}$ " (cf. definition 1.9) is given by:

$$
\theta_{i j}(z)=\exp i\left(\int_{X \times I} T P\left(A_{i}(z), A_{i}^{\tilde{g}_{i j}}(z)\right)+\int_{X} T P\left(A_{0}, A_{i}(z), A_{j}(z)\right)\right) .
$$

Hence the transition functions $\theta_{i j}^{*}$ of $\mathscr{L}^{*}$ are $\theta_{i j}^{*}=\theta_{i j}^{-1}=\theta_{j i}$, or

$$
\theta_{i j}^{*}=\exp i\left(-\int_{X \times I} T P\left(A_{i}, A_{i}^{\tilde{g}_{i j}}\right)-\int_{X} T P\left(A_{0}, A_{i}, A_{j}\right)\right) .
$$

On $U_{i} \cap U_{j}$ we must show the compatibility condition $c s_{j}=c s_{i} \theta_{i j}^{*}$. To see this first consider $c_{0}^{-1} c s_{i} \theta_{i j}^{*}$, where $c_{0}=c s\left(\bar{A}_{0}, \bar{A}_{00}\right)$. Then

$$
\begin{aligned}
c_{0}^{-1} C s_{i}(z) \theta_{i j}^{*}(z)=\exp i\left(-\int_{Y} T P\left(\bar{A}_{0}, \bar{A}_{i}(z)\right)-\int_{X \times I} T P\left(A_{i}(z), A_{i}^{\tilde{g}_{i j}}(z)\right)\right. \\
\left.\quad-\int_{X} T P\left(A_{0}, A_{i}(z), A_{j}(z)\right)\right) \\
=\exp i\left(-\int_{Y} T P\left(\bar{A}_{0}, \bar{A}_{j}(z)\right)+\int_{Y} T P\left(\bar{A}_{i}, \bar{A}_{j}\right)\right. \\
\left.-\int_{X \times I} T P\left(A_{i}(z), A_{i}^{\tilde{g}_{i j}}(z)\right)\right),
\end{aligned}
$$

since

$$
-\int_{X} T P\left(A_{0}, A_{i}, A_{j}\right)=\int_{Y} T P\left(\bar{A}_{0}, \bar{A}_{i}\right)-\int_{Y} T P\left(\bar{A}_{0}, \bar{A}_{j}\right)+\int_{Y} T P\left(\bar{A}_{i}, \bar{A}_{j}\right) .
$$


Claim.

$$
\int_{X \times I} T P\left(A_{i}(z), A_{i}^{\tilde{g}_{i j}}(z)\right)=\int_{Y} T P\left(\bar{A}_{i}(z), \bar{A}_{j}(z)\right) \quad \bmod 2 \pi \mathrm{Z} .
$$

To show this we observe that

$$
-\int_{Y} T P\left(\bar{A}_{i}(z), \bar{A}_{j}(z)\right)=\int_{-Y} T P\left(\bar{A}_{i}(z), \bar{A}_{j}(z)\right),
$$

where $-Y$ denotes $Y$ with the opposite orientation. Recall that by antisymmetry, $\int_{Y} T P\left(\bar{A}_{i}(z), \bar{A}_{i}(z)\right)=0$. Then consider the closed $(2 k-1)$-manifold $W=Y \cup_{X \times\{0\}}(X \times I) \cup_{X \times\{1\}}(-Y)$, where a connection $B$ can be defined as

$$
B(z)= \begin{cases}\bar{A}_{i}(z) & \text { on } Y \\ A_{i}^{\tilde{g}_{i j}}(z) & \text { on } X \times I \\ \bar{A}_{j}(z) & \text { on }-Y\end{cases}
$$


FIgURE 1. $W=Y \cup_{X \times\{0\}}(X \times I) \cup_{X \times\{1\}}(-Y)$

The problem now reduces to showing that

$$
\int_{W} T P\left(\bar{A}_{i}(z), B(z)\right)=0 \quad \bmod 2 \pi \mathrm{Z}
$$

By earlier remarks there exists a gauge transformation $\bar{g}_{i j}$ on $\overline{\mathscr{P}}$ such that $\bar{A}_{i}^{\bar{g}_{i j}}=\bar{A}_{j}$. Then there is a gauge transformation $h$ given by

$$
h= \begin{cases}\text { id } & \text { on } Y \\ \tilde{g}_{i j} & \text { on } X \times I \\ \bar{g}_{i j} & \text { on }-Y\end{cases}
$$

such that $B=\bar{A}_{i}^{h}$. With this the integral in (3.9) reads

$$
\int_{W} T P\left(\bar{A}_{i}(z), \bar{A}_{i}^{h}(z)\right)
$$


or, with the definition of $T P$

$$
2 \pi \int_{W} \int_{s=0}^{1} P\left(F_{(1-s) \bar{A}_{i}(z)+s \bar{A}_{i}^{h}(z)}\right) .
$$

Now, at the ends of the manifold $W \times I$, the differential form under the integral are $P\left(F_{\bar{A}_{i}(z)}\right)$ and $P\left(F_{\bar{A}_{i}^{h}(z)}\right)$ respectively, but since we are dealing with forms on the base, these two forms agree. Again we apply the mapping torus of $\overline{\mathscr{P}}$. This is $\overline{\mathscr{P}} \times I / \sim$, where $(p, 1) \sim(h \cdot p, 0)$, which on the base is just $W \times I / \sim$ with $(w, 1) \sim(w, 0)$. In other words the integral in (3.10) can be rewritten as

$$
\int_{W \times S^{1}} P\left(F_{(1-s)} \bar{A}_{i}(z)+s \bar{A}_{i}^{h}(z)\right),
$$

which is integer valued. This concludes the proof of the compatibility condition.

Next we show that the section does not depend on the extension $\bar{A}_{0}$ of the connection $A_{0}$. Let $A_{0}$ be a connection in $\mathscr{P}$. Let $\bar{A}_{0}$ and $\bar{A}_{0}^{\prime}$ be two connections in $\overline{\mathscr{P}}$ extending $A_{0}$. Let $c s_{i}$ and $c s_{i}^{\prime}$ be defined over $U_{i}$ by

$$
c s_{i}(z)=c_{0} \exp i \int_{Y} T P\left(\bar{A}_{0}, \bar{A}_{i}(z)\right), \quad c s_{i}^{\prime}(z)=c_{0}^{\prime} \exp i \int_{Y} T P\left(\bar{A}_{0}^{\prime}, \bar{A}_{i}(z)\right)
$$

with $c_{0}=\operatorname{cs}\left(\bar{A}_{0}, \bar{A}_{00}\right), c_{0}^{\prime}=\operatorname{cs}\left(\bar{A}_{0}^{\prime}, \bar{A}_{00}\right)$. Then

$$
\begin{aligned}
\left(c_{0}^{-1} c s_{i}(z)\right)^{-1} c_{0}^{\prime} c s_{i}^{\prime}(z) & =\exp i\left(\int_{Y} T P\left(\bar{A}_{0}, \bar{A}_{i}(z)\right)-\int_{Y} T P\left(\bar{A}_{0}^{\prime}, \bar{A}_{i}(z)\right)\right) \\
& =\exp i\left(\int_{Y} T P\left(\bar{A}_{0}, \bar{A}_{0}^{\prime}\right)+\int_{X} T P\left(A_{0}, A_{0}, A_{i}(z)\right)\right) \\
& =\exp i \int_{Y} T P\left(\bar{A}_{0}, \bar{A}_{0}^{\prime}\right) .
\end{aligned}
$$

On the other hand, by (3.1) we have

$$
\begin{aligned}
c_{0}\left(c_{0}^{\prime}\right)^{-1}=\exp \left(2 \pi i\left\langle S_{P}(\bar{B})-S_{P}\left(\bar{B}^{\prime}\right),[W]\right\rangle\right) & \\
& \exp \left(-i \int_{W} T P\left(\bar{B}, \bar{B}^{\prime}\right)\right)=\exp \left(-i \int_{Y} T P\left(\bar{A}_{0}, \bar{A}_{0}^{\prime}\right)\right) .
\end{aligned}
$$

by [9] (2.10). This proves that $c s$ is a well-defined section. Finally we calculate the covariant derivative of the section with respect to the connection obtained 
in section 2. Locally we have, using the formula for $c s_{i}$ with $c_{0}=\operatorname{cs}\left(\bar{A}_{0}, \bar{A}_{00}\right)$ :

$$
\begin{aligned}
d c s_{i}= & c_{0} d \exp \left(-i \int_{Y} T P\left(\bar{A}_{0}, \bar{A}_{i}\right)\right) \\
= & -i d_{Z} \int_{Y} T P\left(\bar{A}_{0}, \bar{A}_{i}\right) \otimes c s_{i} \\
= & -2 \pi i \int_{Y} \int_{s=0}^{1} d_{Z} P\left(F_{\left.(1-s) \bar{A}_{0}+s \bar{A}_{i}\right) \otimes c s_{i}}\right. \\
= & -2 \pi i \int_{Y} \int_{s=0}^{1}\left(d-d_{Y}-d_{s}\right) P\left(F_{\left.(1-s) \bar{A}_{0}+s \bar{A}_{i}\right) \otimes c s_{i}}\right. \\
= & 0-i \int_{X} T P\left(A_{0}, A_{i}\right) \otimes s_{i}-2 \pi i \int_{Y} P\left(F_{\bar{A}_{0}}\right) \otimes c s_{i} \\
& \quad+2 \pi i \int_{Y} P\left(F_{\bar{A}_{i}}\right) \otimes c s_{i} .
\end{aligned}
$$

Here the form $2 \pi i \int_{Y} P\left(F_{\bar{A}_{0}}\right)$ is zero, since it does not depend on $z \in Z$. On the other hand, the one-form $\omega_{i}$ that determines the connection of $\mathscr{L}$ was given by

$$
\omega_{i}=-i \int_{X} T P\left(A_{0}, A_{i}\right)
$$

cf. definition 2.3. Hence the connection of $\mathscr{L}^{*}$ is determined by the one-form

$$
\omega_{i}^{*}=i \int_{X} T P\left(A_{0}, A_{i}\right)
$$

It follows that

$$
\nabla\left(c s_{i}\right)=\omega_{i}^{*} \otimes c s_{i}+d c s_{i}=2 \pi i \int_{Y} P\left(F_{\bar{A}_{i}}\right) \otimes c s_{i},
$$

and hence, globally

$$
\nabla(c s)=2 \pi i \int_{Y} P\left(F_{\bar{A}}\right) \otimes c s .
$$

REMARK 3.13. The Chern-Simons section of the relative line bundle from remark 1.27 is given locally by $c s_{i}^{A B}(z)=\exp \left(-i \int_{Y} T P\left(\bar{B}_{i}(z), \bar{A}_{i}(z)\right)\right.$, and its covariant derivative is $\nabla^{A B}\left(c s_{i}^{A B}\right)=2 \pi i\left(\int_{Y} P\left(F_{\bar{A}_{i}}\right)-\int_{Y} P\left(F_{\bar{B}_{i}}\right)\right) \otimes c s_{i}^{A B}$. 


\section{Application to handle bodies}

As an example we apply our results to a family of flat connections over a genus $g$ surface $X$. We shall show the following:

Proposition 4.1. Let $X$ be a genus $g$ surface and $Y$ the corresponding handle body such that $\partial Y=X$. Let $\rho_{z}: \pi_{1}(Y) \rightarrow S U(2)$ be a family of representations of the fundamental group of $Y$ indexed by a manifold $Z$ and choose the Maurer-Cartan connection in the trivial bundle over $Y$ as boundary condition in the sense of 3.2(5). Then these data define a line bundle $\mathscr{L} \rightarrow Z$ with a canonical flat connection and an everywhere non-zero Chern-Simons section which is parallel.

Proof. Let $Y$ be the "massive interior" of $X$ such that $\partial Y=X$. The homotopy type of $Y$ is the same as a wedge of $g$ circles, so the fundamental group is the free group with $g$ generators.

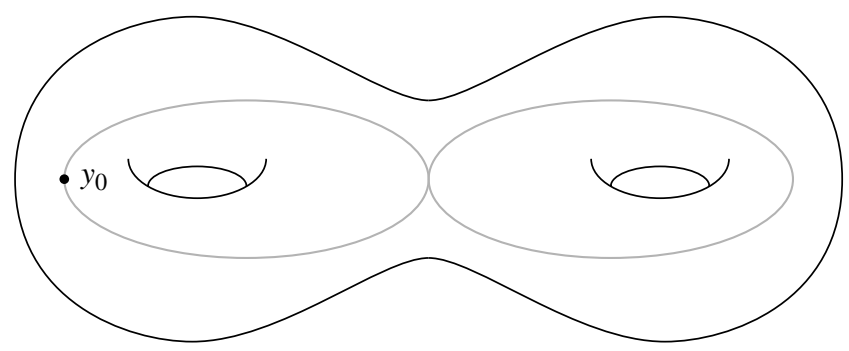

FIGURE 2. A genus 2 handle body with generators of the fundamental group

Let $\tilde{Y}$ be the fundamental covering of $Y$ and $\pi: \tilde{Y} \rightarrow Y$ the projection. Let

$$
\rho: \pi_{1}(Y) \rightarrow S U(2)
$$

be a representation of the fundamental group of $Y$. Let

$$
\tilde{Y} \times{ }_{\rho} S U(2)
$$

be the quotient by the equivalence relation $(\tilde{y} \cdot \xi, g) \sim(\tilde{y}, \rho(\xi) g)$ for $\xi \in$ $\pi_{1}(Y)$. This is a principal $S U(2)$-bundle over $Y$; it is well-known that it is trivial. In particular it makes sense to use the Maurer-Cartan connection as "boundary condition" in the sense of 3.2 (5). A set of representations $\left.\left\{\rho_{z}\right\}\right|_{z \in Z}$ determine a family of (trivial) principal $S U(2)$-bundles $\mathscr{P}_{z}$ and hence a line bundle $\mathscr{L} \rightarrow Z$, cf. theorem 1.3. Now let $U$ be a plane disc with $g$ holes and note that there is a deformation retraction

$$
r: Y \rightarrow U \text {. }
$$


Then the above family of flat principal bundles is induced from $U$ via $r$, more precisely there is a deformation retraction $\bar{r}: Z \times Y \rightarrow Z \times U$ such that a bundle $\bar{E} \rightarrow Z \times Y$ as the one in theorem 3.3 is the pull back via $\bar{r}$ from a bundle $\tilde{E} \rightarrow Z \times U$. The form $P\left(F_{\bar{A}}\right)$ on $Y$ in (3.12) is the pull-back of a form on $U$, and hence the integral

$$
\int_{Y} P\left(F_{\bar{A}}\right)
$$

in (3.12) is zero. It follows that the Chern-Simons section $c s$ is parallel, and the connection has zero curvature.

\section{REFERENCES}

1. Bonora, L., Cotta-Ramusino, P., Rinaldi, M., Stasheff, J., The evaluation map in field theory, sigma-models and strings -II, Comm. Math. Phys. 114 (1988), 381-438.

2. Brylinski, J. -L., Geometric construction of Quillen line bundles, in Advances in Geometry, ed. J. -L. Brylinski, Progr. Math. 172, 1999.

3. Brylinski, J. L., Loop Spaces, Characteristic Classes and Geometric Quantization, Progr. Math. 107, 1993.

4. Bitmut, J. M., Freed, D., The analysis of elliptic families I: Metrics and connections on determinant bundles, Comm. Math. Phys. 106 (1986), 159-176.

5. Bott, R., Tu, L. W., Differential Forms in Algebraic Topology, Grad. Texts in Math. 82, 1982.

6. Cheeger, J., Simons, J., Differential characters and geometric invariants, in Geometry and Topology, Proc. Spec. Year, College Park/Md. 1983/84, eds. J. Alexander and J. Harer, Lecture Notes in Math. 1167, 1985, 50-80.

7. Chern, S. S., Simons, J., Characteristic forms and geometric invariants, Ann. of Math. 99 (1974), 48-69.

8. Dai, X., Freed, D., Eta-invariants and determinant lines, J. Math. Phys. 35 (1994), 5155-5194.

9. Dupont, J. L., Kamber, F., On a generalization of Cheeger-Chern-Simons classes, Illinois J. Math. 34 (1990), 221-255.

10. Freed, D., On determinant line bundles, in Mathematical Aspects of String Theory, ed. S. T. Yau, World Scientific Publishing, 1987, pp. 189-238.

11. Freed, D., Determinant line bundles revisited, in Proceedings of the conference Geometry and Physics, Aarhus, Denmark, July 18-27, 1995, ed. J. E. Andersen et. al., Lecture Notes in Pure and Appl. Math. 184, Marcel Dekker, Inc., pp. 187-196.

12. Hirzebruch, F., Topological Methods in Algebraic Geometry, 3rd ed., Springer-Verlag, 1978

13. Quillen, D., Determinants of Cauchy-Riemann operators over a Riemann surface, Funct. Anal. Appl. 19, No. 1 (1985), 31-34.

14. Ramadas, T. R., Singer, I. M., Weitsman, J., Some comments on Chern-Simons gauge theory, Comm. Math. Phys. 126 (1989), 409-420.

DEPARTMENT OF MATHEMATICS

UNIVERSITY OF AARHUS

BYGNING 530

8000 ÅRHUS C

DENMARK

E-mail: dupont@imf.au.dk
IT UNIVERSITY OF COPENHAGEN

GLENTEVEJ 67

2400 KØBENHAVN NV

DENMARK

E-mail: lindblad@krypton.dk 